\title{
RESÍDUOS ORGÂNICOS AGROINDUSTRIAIS INFLUENCIAM A PRODUÇÃO DE HÚMUS E A MULTIPLICAÇÃO DE MINHOCAS
}

\author{
AGRO-INDUSTRIAL ORGANIC RESIDUE INFLUENCE THE PRODUCTION OF HUMUS \\ AND MULTIPLICATION OF EARTHWORMS \&
}
LOS RESIDUOS ORGÁNICOS AGROINDUSTRIALES INFLUYEN EN LA PRODUCCIÓN DE HÚMUS Y LA MULTIPLICACIÓN DE LOMBRICES DE TIERRA \&

Recebido em: 11/10/2021 - Aprovado em: 25/10/2021 - Publicado em: 07/11/2021

doi) http://dx.doi.org/10.18011/bioeng2021v15n3p401-410

\begin{abstract}
Mayara Camila Soares Santos ${ }^{1}$ (mayara.santos.agro@hotmail.com)
Cleberton Correia Santos ${ }^{2}$ (cleber_frs@yahoo.com.br)

Ivo de Sá Motta 3 (ivomotta@embrapa.br)
\end{abstract}

\footnotetext{
1 Universidade Estadual do Mato Grosso do Sul (UEMS). Dourados, MS, Brasil.

2 Universidade Federal da Grande Dourados (UFGD). Dourados, MS, Brasil.

${ }^{3}$ Empresa Brasileira de Pesquisa Agropecuária (EMBRAPA) - CPAO. Dourados, MS, Brasil.
}

\section{RESUMO}

Dentre as técnicas utilizadas no aproveitamento de resíduos agroindustriais, tal como o bagaço de canade-açúcar e o conteúdo ruminal bovino, a vermicompostagem tem se mostrado uma opção, utilizandose minhocas para a degradação desses resíduos pelo trato digestivo, transformando-os em húmus. A minhoca gigante africana é muito utilizada nesse processo de biotransformação e estabilização desses materiais. Assim, objetivou-se com este trabalho conhecer a viabilidade do uso de bagaço de cana e conteúdo ruminal bovino na produção de húmus e multiplicação de minhocas. Os tratamentos foram constituídos por diferentes proporções de conteúdo ruminal (CR) e bagaço de cana (BC), sendo: ( $\left.T_{1}\right)$ $100 \% \mathrm{CR}$; ( $\left.\mathrm{T}_{2}\right)$ 87,5\% CR + 12,5\% BC; ( $\left.\mathrm{T}_{3}\right) 75 \% \mathrm{CR}+25 \% \mathrm{BC} ;\left(\mathrm{T}_{4}\right) 62,5 \% \mathrm{CR}+37,5 \% \mathrm{BC} ;\left(\mathrm{T}_{5}\right) 50 \%$ $\mathrm{CR}+50 \% \mathrm{BC}$. Realizou-se a inoculação das minhocas (massa inicial de $300 \mathrm{~g} / \mathrm{leira}$ ) no período matutino, e decorridos 78 dias, foi quantificada a massa de minhocas e de húmus. A combinação de $75 \%$ de conteúdo ruminal $+25 \%$ de bagaço de cana favorece a multiplicação de minhocas gigante africana. O teor de $100 \%$ de conteúdo ruminal possibilita a maior produção de húmus, e conforme se adiciona bagaço de cana, é observada uma diminuição de húmus.

Palavras-chave: Bagaço de cana-de-açúcar. Conteúdo ruminal. Eudrilus eugeniae. vermicompostagem.

Artigo publicado sob a licença Creative Commons - Atribuição 4.0 Internacional (CC BY 4.0). 
1 INTRODUÇÃO

Nos últimos anos o setor de agroindústrias tem-se desenvolvido representando importante papel na economia; no entanto, o aumento da produção tem gerado quantidade significativa de resíduos (GASPAR et al., 2020). Em geral, os resíduos resultantes quando não tratados de forma correta, podem causar sérios problemas ambientais, tais como poluição do solo, de águas superficiais e subterrâneas (FURLAN et al., 2018).

Dentre os resíduos orgânicos agroindustriais, destacam-se o conteúdo ruminal proveniente de frigorifico bovino e o bagaço de cana-de-açúcar devido à ampla disponibilidade em diversos estados do Brasil. Desta forma, o emprego de técnicas que possam minimizar e/ou atenuar esses passivos ambientais deve ser elucidado.

O correto aproveitamento de resíduos pode ser uma alternativa para a redução dos custos na compra de substratos comerciais e fontes de adubos orgânicos (ANTUNES et al., 2012; CUNHA et al., 2015), desde que estejam estabilizados. Visando a bioestabilização desses materiais orgânicos, a vermicompostagem é umas das técnicas utilizadas.

A vermicompostagem consiste na reciclagem de materiais orgânicos pelo trato disgestivo de minhocas, excretando os coprólitos (VILLEGAS-CORNELIO; CANEPA, 2017; NADOLNY et al., 2020), conhecido como húmus. O húmus de minhoca é considerado um material de elevada qualidade, podendo ser utilizado na forma de adubo orgânico, substrato ou acrescentado a biofertilizantes por ser rico em macro e micronutrientes. A criação de minhocas é altamente viável devido ao baixo investimento e potencial retorno econômico (FILHO et al., 2005; VILLEGAS-CORNELIO; CANEPA, 2017).

A minhoca gigante africana (Eudrilus eugeniae) é uma espécie muito utilizada na bioestabilização desses materiais e apresenta alta capacidade de adaptação ao clima tropical (MORAES et al., 2012), apresentando ainda potencialidade de comerciaização. Desta forma, é de suma relevância a multiplicação dessa espécie.

Todavia, são incipientes os estudos científicos na literatura sobre a importância do aproveitamento resíduos orgânicos agroindustriais (BAHT et al., 2015; CAl et al., 2020) e da minhoca gigante africana na produção de húmus. Assim, objetivou-se com este trabalho conhecer a viabilidade do uso de diferentes combinações de bagaço de cana-de-açúcar e conteúdo ruminal bovino na multiplicação de minhocas e produção de húmus. 
O experimento foi desenvolvido no ano de 2013, no Campus Experimental da

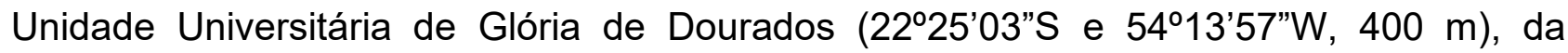
Universidade Estadual de Mato Grosso do Sul, Mato Grosso do Sul, Brasil. O clima da região é do tipo Aw, segundo a classificação de Köppen-Geiger, caracterizando-se por clima tropical chuvoso com inverno seco (ALVARES et al., 2013).

Avaliaram-se cinco combinações de resíduos orgânicos na produção de húmus e multiplicação de minhocas, sendo: $T_{1}-100 \%$ de conteúdo ruminal $(C R) ; T_{2}-87,5 \%$ (CR) $+12,5 \%$ de bagaço de cana (BC); $\mathrm{T}_{3}-75 \% \mathrm{CR}+25 \% \mathrm{BC} ; \mathrm{T}_{4}-62,5 \% \mathrm{CR}+37,5 \% \mathrm{BC} ; \mathrm{T}_{5}$ - $50 \%$ CR $+50 \%$ BC. O bagaço-de-cana e o conteúdo ruminal foi proveniente de usina sucroenergética e frigorífico bovino do estado de Mato Grosso do Sul, respectivamente. O delineamento experimental utilizado foi o inteiramente casualizado, com quatro repetições. A unidade experimental foi constituída por parcelas nas dimensões de $0,60 \mathrm{~m}$ de largura $x$ $0,50 \mathrm{~m}$ de altura $\times 2,5 \mathrm{~m}$ de comprimento, com volume inicial de 600 litros.

Decorridos 15 dias após o preparo das leiras com os tratamentos acima descritos, realizou-se a inoculação das minhocas (a massa inicial de minhocas foi de $300 \mathrm{~g} / \mathrm{unidade}$ experimental), no período das 7 às $9 \mathrm{~h}$. Durante a execução do experimento, foram realizadas irrigações diárias visando manter a umidade em torno de 60 a $70 \%$ da capacidade de campo, utilizando-se sistema do tipo gotejamento; também foi feita a retirada de plantas espontâneas de maneira manual sobre e entre as leiras.

Aos 78 dias após a inoculação das minhocas, foram retiradas amostras de húmus para análise química de macronutrientes de acordo com a metodologia proposta por Silva (2009), e quantificou-se a massa final $(\mathrm{kg})$ de húmus e minhocas dos tratamentos correspondentes. As minhocas foram contadas manualmente e a massa final estipulada em balança de precisão.

Os dados obtidos foram submetidos à análise de variância, e quando significativos pelo teste $\mathrm{F}$ as médias foram submetidas à análise de regressão (significância dos modelos matemáticos linear ou quadrático empregados e coeficiente de determinação $-R^{2}>0,60$ ), a $1 \%$ de probabilidade, utilizando-se o software SISVAR. 


\section{RESULTADOS E DISCUSSÃO}

A composição química do húmus de minhoca (Tabela 1) variou de forma discreta em função das combinações dos resíduos orgânicos. O maior teor de $\mathrm{N}$ e Ca foi observado na formulação de $87,5 \% \mathrm{CR}+12,5 \% \mathrm{BC}$. Já, o maior teor de $\mathrm{P}$ ocorreu na combinação de $75 \% \mathrm{CR}+25 \% \mathrm{BC}$, e $\mathrm{K}$ com $50 \% \mathrm{CR}+50 \% \mathrm{BC}$.

Tabela 1 - Caracterização química das combinações de bagaço de cana e conteúdo ruminal aos 78 dias (após a inoculação das minhocas).

\begin{tabular}{cccccc}
\hline Combinações $^{*}$ & \multicolumn{5}{c}{ Macronutrientes $\left(\mathrm{g} \mathrm{kg}^{-1}\right)$} \\
\cline { 2 - 6 } & $\mathrm{N}$ & $\mathrm{P}$ & $\mathrm{K}$ & $\mathrm{Ca}$ & $\mathrm{Mg}$ \\
\hline $\mathrm{T}_{1}$ & 12,90 & 3,10 & 0,40 & 7,90 & 0,40 \\
$\mathrm{~T}_{2}$ & 16,80 & 3,80 & 0,50 & 13,70 & 0,40 \\
$\mathrm{~T}_{3}$ & 13,90 & 4,00 & 0,50 & 10,20 & 0,40 \\
$\mathrm{~T}_{4}$ & 10,60 & 3,20 & 0,50 & 8,50 & 0,50 \\
$\mathrm{~T}_{5}$ & 15,57 & 3,30 & 0,60 & 8,10 & 0,50 \\
\hline
\end{tabular}

${ }^{*} \mathrm{~T}_{1}-100 \% \mathrm{CR} ; \mathrm{T}_{2}-87,5 \% \mathrm{CR}+12,5 \% \mathrm{BC} ; \mathrm{T}_{3}-75 \% \mathrm{CR}+25 \% \mathrm{BC} ; \mathrm{T}_{4}-62,5 \% \mathrm{CR}+37,5 \% \mathrm{BC} ; \mathrm{T}_{5}-50 \%$ $C R+50 \% B C$.

Fonte: Autores.

A massa final de minhocas e de húmus foi influenciada $(p<0,01)$ pelas combinações dos resíduos agroindustriais. Observa-se que a maior massa de minhocas $(2,57 \mathrm{~kg})$ foi obtida na combinação de $75 \%$ conteúdo ruminal $+25 \%$ de bagaço de cana (Figura 1 ).

Verificamos que a adição de bagaço de cana favoreceu as características físicas, tal como porosidade do húmus. Isso, porque o bagaço de cana apresenta densidade média de $150 \mathrm{~kg} \mathrm{~m}^{-3}$ e umidade em torno de 70 a $80 \%$ (SOUZA et al., 2016), ou seja, quando adicionado, faz com que os húmus apresentem melhores características físicas, tal como aeração, tornando-o favorável a abertura de galerias (BROWN et al., 2006; BROWN, 2007; ROSSI et al., 2006; JAMES, 2007), facilitando a movimentação das minhocas para as camadas mais profundas devido ao caráter fotofóbico (STEFFEN et al., 2013); consequentemente propiciando a multiplicação, como observado nesse estudo. 
Figura 1 - Massa de minhocas gigante africana em função de combinações de resíduos agroindustriais.

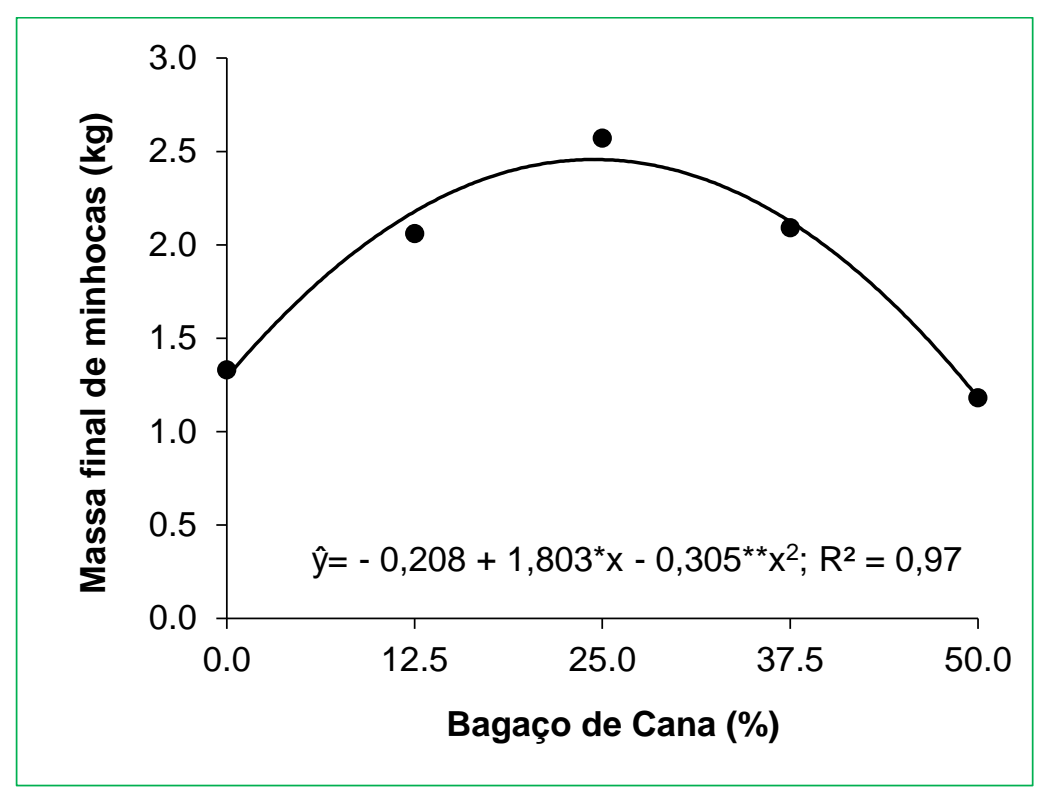

Fonte: Autores

Para a massa final de húmus, o maior valor $(108,90 \mathrm{~kg})$ foi verificado na combinação de $100 \%$ de conteúdo ruminal (Figura 2). Conforme se adiciona maiores proporções de bagaço de cana, a massa final é reduzida. Provavelmente, a adição crescente do bagaço de cana ao conteúdo ruminal pode ter favorecido o desenvolvimento de micro-organismos em função da melhor estabilidade de temperatura e umidade, e consequentemente tenham tornado o material uma fonte alimentar mais acessível às minhocas (AQUINO et al., 1994), reduzindo assim a massa final de húmus. 
Figura 2 - Massa de húmus aos 78 dias após inoculação de minhocas gigante africana em função de combinações de resíduos agroindustriais.

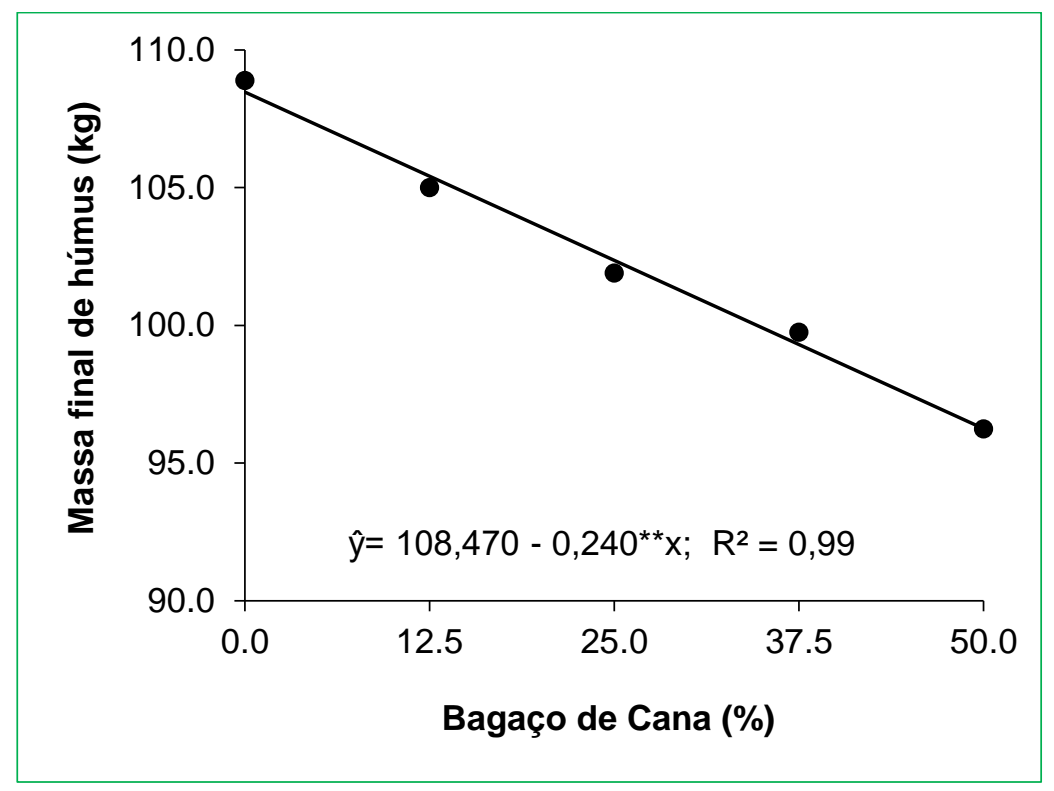

Fonte: Autores

Corroborando com nossos resultados, Dores-Silva et al. (2013) observaram ao estudar o processo de vermicompostagem com diferentes resíduos orgânicos, que houve elevado grau de humificação e redução da relação $\mathrm{C} / \mathrm{N}$. A vermicompostagem é importante na estabilização dos resíduos orgânicos, tendo em vista que esse processo representa a conversão desses materiais, tais como os estudados nesse trabalho, originando em composto rico em ácidos fúlvicos, húmicos e humina que são as frações da matéria orgânica com alto grau de estabilização (BALDOTTO \& BALDOTTO, 2014; SILVA et al., 2015); além de ser rico em macronutrientes (Tabela 1).

\section{CONCLUSÕES}

O uso de resíduos agroindustriais é víavel para a vermicomostagem, e a combinação de $75 \%$ de conteúdo ruminal bovino $+25 \%$ de bagaço de cana-de-açúcar propicia melhores condições para multiplicação de minhocas gigante africana. Conforme se adiciona bagaço de cana, é observada diminuição na produção de húmus. 


\section{REFERÊNCIAS}

ALVARES, C. A.; STAPE, J. L.; SENTELHAS, P. C.; GONÇALVES, J. L. M.; SPAROVERK, G. Köppen's climate classification map for Brazil. Meteorologische Zeitschrift, v. 22, n. 6, p. 711-728, 2013.

ANTUNES, R. M.; CASTILHOS, R. M. V.; CASTILHO, D. D.; LEAL, O. A.; ANDREAZZA, $R$. Crescimento inicial de acácia negra com vermicomposto de diferentes resíduos agroindustriais. Ciência Florestal, v. 26, n. 1, p. 1-9, 2012.

AQUINO, A. M.; ALMEIDA, D. L.; FREIRE, L. R.; DE-POLLI, H. Reprodução de minhocas (Oligochaeta) em esterco bovino e bagaço de cana de açúcar. Pesquisa Agropecuária Brasileira, v. 29, n. 2, p. 161-168, 1994.

BALDOTTO, M. A.; BALDOTTO, L. E. B. Ácidos húmicos. Revista Ceres, v. 61, sumplemento, p. 856-881, 2014.

BHAT, S. A.; SINGH, J.; VIG, A. P. Potential utilization of bagasse as feed material for earthworm Eisenia fetida and production of vermicompost. Springer Plus, v. 4, n. 11, p. 19, 2015.

BROWN, G. G.; JAMES, S. W. Ecologia, biodiversidade e biogeografia das minhocas no Brasil. In: BROWN, G. G.; FRAGOSO, C. (Ed.) - Minhocas na América Latina: Biodiversidade e ecologia. Londrina, Embrapa Soja, p. 297-381, 2007.

BROWN, G. G.; JAMES, S. W.; PASINI, A.; NUNES, D. H.; BENITO, N. P.; MARTINS, P. T. E.; SAUTTER, K. D. Exotic, peregrine, and invasive earthworms in Brazil: diversity, distribution, and effects on soils and plants. Caribbean Journal of Science, v. 42, p. 339358, 2006.

CAI, L.; SUN, X.; HAO, D.; LI, S.; GONG, X.; YU, K. Sugarcane bagasse amendment improves the quality of green waste vermicompost and the growth of Eisinia fetida. Frontiers of Environmental Science \& Engineering, v. 14, n. 4, p. 1-11, 2020.

CUNHA, A. H. N.; BRASIL, E. P. F.; FERREIRA, R. B.; VIEIRA, J. A.; ARAÚJO, C. S. T.; SILVA, S. M. C. Vermicompost of tannery sludge and sewage as conditioners soil with grown tomato. African Journal of Agricultural Research, v. 11, p. 4086-4091, 2015.

DORES-SILVA, P. R., LANDGRAF, M. D., REZENDE, M. O. O. Processo de estabilização de resíduos orgânicos: vermicompostagem versus compostagem. Química Nova, v. 36, n. 5, p. 640-645, 2013.

FILHO, D. O., FERRAZ, I. D., MARTINS, J. H., SANTOS, L. C., FILHO, O. P. R., COSTA, D. R. 2005. Avaliação do deslocamento de minhocas (Eudrilus eugeniae) submetidas a pulsos elétricos controlados. Revista Brasileira de Engenharia Agrícola e Ambiental, v. 9, n. 3, p. 433-440, 2005. 
FURLAN, F. L.; CONSOLIN FILHO, N.; CONSOLIN, M. F. B.; GONÇALVES, M. S.; VALDERRAMA, P.; GENENA, A. K. Use of agricultural and agroindustrial residues as alternative adsorbents of manganese and iron in aqueous solution. Ambiente \& Água, v. 13, n. 2, p. 1-12, 2018.

GASPAR, L. M. R.; INÁCIO, C. T.; QUINTAES, B. R.; CARVALHO, L. S. Q.; PERES, A. A. C. Análise econômica-financeira do gerenciamento dos resíduos sólidos orgânico em uma agroindústria de processamento mínimo de hortaliças. Engenharia Sanitária e Ambiental, v. 25, n. 3, p. 477-488, 2020.

MORAES, M. J., FILHO, D. O., MARTINS, J. H., SANTOS, L. C. 2012. Electric signals for separation of earthworms (Eudrilus eugeniae). Revista Brasileira de Engenharia Agrícola e Ambiental, v. 16, n. 3, p. 1137-1142, 2012.

NADOLNY, H.; SANTOS, A.; DEMETRIO, W. FERREIRA, T.; MAIA, L. S.; CONRADO, A. C.; BARTZ, M.; GARRASTAZU, M.; SILVA, E. LAVELLE, P.; BARETTA, D.; PASINI, A.; VEZZANI, F.; SOUSA, J. P.; CUNHA, L.; MATHIEU, J.; RÖMBKE, J.; BROWN, G. Recommendations for assessing earthworm populations in Brazilian ecosystems. Pesquisa Agropecuária Brasileira, v. 55, e01006, p. 1-35, 2020.

PEEL, M. C., FINLAYSON, B. L., MCMAHON, T. A. Updated world map of the KöppenGeiger climate classification. Hydrology and Earth System Sciences, v. 11, p. 16331644, 2007.

ROSSI, J. P., HUERTA, E., FRAGOSO, C., LAVELLE, P. Soil properties inside earthworm patches and gaps in a tropical grassland (La Mancha, Veracruz, Mexico). European Journal of Soil Biology, v. 42, n. 1, p. 284-288, 2006.

SILVA, F. C. Manual de análises químicas do solo, plantas e fertilizantes. 2. ed. rev. ampliada-Brasília, DF: Embrapa Informação Tecnológica, 2009. 627 p.

SILVA, M. A. C., SANTOS, W. O., SIMOURA, N. T., TESCH, J. A., RUAS, K. F., COlOdeTE, C. M., TANNURE, F. P., BARBIRATO, J. O., RAMOS, A. C., DOBBSS, L. B. Ácidos húmicos de vermicomposto estimulam o crescimento in vitro de plântulas de Cattey awarneri (Orchidaceae). Rodriguésia, v. 66, n. 3, p. 759-768, 2015.

SOUZA, C. F., CIRILO, L. S., BASTOS, R. G., PERES, J. G., OLIVEIRA, A. F. 2016. Sonda de TDR para a estimativa de umidade em bagaço de cana de açúcar.

Engenharia Agrícola, v. 36, n. 1, p. 24-35, 2016.

STEFFEN, G. P. K., ANTONIOLLI, Z. I., STEFFEN, R. B., JACQUES, R. J. S. Importância ecológica e ambiental das minhocas. Revista de Ciências Agrárias, v. 36, n. 2, p. 37-147, 2013.

VILLEGAS-CORNELIO, V. M.; CANEPA, J. R. L. Vermicompostaje: Il avances y estrategias em el tratamiento de residuos sólidos orgánicos. Revista Mexicana de Ciencias Agrícolas, v. 8, n. 2, p. 407-421, 2016. 


\begin{abstract}
Among the techniques used in the use of agro-industrial residues, such sugarcane bagasse and bovine ruminal content, vermicomposting has shown itself an option, using earthworms for the degradation of these residues by the digestive tract, transforming in humus. The "gigante africana" earthworms is widely used in this process of biotransformation and stabilization of these materials. Thus, the aim of this work was to know the feasibility of use sugarcane bagasse and bovine ruminal content in the production of humus and multiplication of earthworm. The treatments were constituted by different ratios of ruminal content (RC) and sugarcane bagasse (SB), being: $\left.\mathrm{T}_{1}\right) 100 \% \mathrm{RC}$; $\mathrm{T}_{2}$ ) $87.5 \% \mathrm{RC}+12.5 \%$ $\mathrm{SB}$; $\left.\mathrm{T}_{3}\right) 75 \% \mathrm{RC}+25 \% \mathrm{SB}$; $\left.\left.\mathrm{T}_{4}\right) 62.5 \% \mathrm{RC}+37.5 \% \mathrm{SB} ; \mathrm{T}_{5}\right) 50 \% \mathrm{RC}+50 \% \mathrm{SB}$. The earthworm was inoculated (initial mass of $300 \mathrm{~g} / \mathrm{leira}$ ) in the morning, and after 78 days, the mass earthworm and humus were quantified. The combination of $75 \%$ ruminal content + $25 \%$ sugarcane favors the multiplication of "gigante africana" eartworm. The content of $100 \%$ ruminal content allows the highest production of humus, and as sugarcane bagasse is added, a decrease in humus is observed.
\end{abstract}

Keywords: Sugarcane bagasse. Ruminal content. Eudrilus eugeniae. Vermicomposting.

\title{
RESUMEN
}

Entre las técnicas utilizadas en el uso de residuos agroindustriales, como el bagacillo de caña de azúcar y el contenido del rumen bovino, se ha demostrado que el vermicompostaje es una opción, utilizando lombrices de tierra para la degradación de estos residuos por el tracto digestivo, convirtiéndolos en humus. Las lombrices de tierra gigante africana son ampliamente utilizadas en este proceso de biotransformación y estabilización de estos materiales. Así, el objetivo de este trabajo fue conocer la viabilidad del uso del bagacillo de caña de azúcar y el contenido de rumen bovino en la producción de humus y multiplicación de las lombrices de tierra. Los tratamientos consistieron en diferentes proporciones de contenido ruminal (CR) y bagacillo de caña de azúcar (BC): $\left(\mathrm{T}_{1}\right) 100 \% \mathrm{CR}$; $\left(\mathrm{T}_{2}\right)$ 87,5\% CR $+12,5 \%$ BC; $\left(\mathrm{T}_{3}\right) 75 \% \mathrm{CR}+25 \% \mathrm{BC}$; ( $\left.\mathrm{T}_{4}\right) 62,5 \% \mathrm{CR}+37,5 \% \mathrm{BC}$; ( $\left.\mathrm{T}_{5}\right) 50 \% \mathrm{CR}+50 \% \mathrm{BC}$. Las lombrices fueron inoculadas (masa inicial de $300 \mathrm{~g} / \mathrm{leira}$ ) por la mañana, y después de 78 días, se cuantificó la masa de lombrices de tierra y humus. La combinación de $75 \%$ de contenido ruminal $+25 \%$ de bagacillo de caña de azúcar favorece la multiplicación de lombrices de tierra gigantes africanas. El contenido de $100 \%$ del contenido de ruminal permite la mayor producción de humus, y a medida que se agrega bagacillo de caña de azúcar, se observa una disminución en el humus.

Palabras clave: Bagacillo de caña de azúcar. Contenido ruminal. Eudrilus eugeniae. Vermicompostaje. 


\section{LICENÇA DE USO}

Este é um artigo publicado em acesso aberto (Open Access) sob a licença Creative Commons Atribuição 4.0 Internacional (CC BY 4.0), que permite uso, distribuição e reprodução em qualquer meio, desde que o trabalho original seja corretamente citado. Mais informações em: http://creativecommons.org/licenses/by/4.0

\section{CONFLITO DE INTERESSES}

Os autores declaram que não há conflito de interesses neste trabalho.

\section{CONTRIBUIÇÕES AUTORAIS}

Autor 1: Instalação, condução, análise estatística e redação científica.

Autor 2: Instalação, condução, análise estatística e redação científica.

Autor 3: Planejamento e redação científica.

\section{FINANCIAMENTO}

O presente trabalho foi realizado com apoio da Empresa Brasileira de Pesquisa Agropecuária (EMBRAPA).

\section{AGRADECIMENTO}

Ao Conselho Nacional de Desenvolvimento Científico e Tecnológico (CNPq), pela concessão da bolsa, à Fundação de Apoio ao Desenvolvimento de Ensino, Ciência e Tecnologia do Estado de Mato Grosso do Sul (FUNDECT) e à Empresa Brasileira de Pesquisa Agropecuária (EMBRAPA), pelo apoio financeiro.

\section{COMO REFERENCIAR}

SANTOS, Mayara Camila Soares; SANTOS, Cleberton Correia; MOTTA, Ivo de Sá.

Resíduos orgânicos agroindustriais influenciam a produção de húmus e a multiplicação de minhocas. Revista Brasileira de Engenharia de Biossistemas (Tupã), v. 15, n. 3, p. 401-410, 2021. DOI: http://dx.doi.org/10.18011/bioeng2021v15n3p401-410.

\section{RESPONSABILIBADE EDITORIAL}

Prof. Dr. Fernando Ferrari Putti ${ }^{1}$, Prof. Dr. Paulo Sérgio Barbosa dos Santos ${ }^{1}$, Prof. Dr. Eduardo Festozo Vicente ${ }^{1}$ e Prof. Dr. Diogo de Lucca Sartori ${ }^{1}$

1 Universidade Estadual Paulista "Júlio de Mesquita Filho", FCE - Faculdade de Ciências e Engenharia, Tupã, SP, Brasil. 